\title{
Simplicial models for trace spaces II: General higher dimensional automata
}

\author{
MARTIN RAUSSEN
}

\begin{abstract}
Higher Dimensional Automata (HDA) are topological models for the study of concurrency phenomena. The state space for an HDA is given as a pre-cubical complex in which a set of directed paths (d-paths) is singled out. The aim of this paper is to describe a general method that determines the space of directed paths with given end points in a pre-cubical complex as the nerve of a particular category.

The paper generalizes the results from Raussen $[18 ; 19]$ in which we had to assume that the HDA in question arises from a semaphore model. In particular, important for applications, it allows for models in which directed loops occur in the processes involved.
\end{abstract}

55P10, 55P15, 55U10; 68Q85, 68Q55

\section{Introduction}

\subsection{Background}

A particular model for concurrent computation in computer science, called Higher Dimensional Automata (HDA), was introduced by Pratt [15] back in 1991. Mathematically, HDA can be described as (labelled) pre-cubical sets (with $n$-dimensional cubes instead of simplices as building blocks; cf Brown and Higgins [2; 1]) with a preferred set of directed paths (respecting the natural partial orders) in any of the cubes of the model.

Compared to other well-studied concurrency models like labelled transition systems, event structures, Petri nets, etc (for a survey on those, see Winskel and Nielsen [23]), R J van Glabbeek [22] showed that Higher Dimensional Automata have the highest expressivity; on the other hand, they are certainly less studied and less often applied so far.

All concurrency models deal with sets of states and with associated sets of execution paths (with some further structure). The interest is mainly in the structure of the spaces of execution paths; typically, it is difficult to extract valuable information about the path 
space from the state space model. We use topological models for both state space and the execution (=path) space consisting of the directed paths (called d-paths) in state space. It is particularly important to know whether the path space is path-connected; and, if not, to get an overview over its path components: Executions in the same path component yield the same result (decision) in a concurrent computation; different components may lead to different results. From a topological perspective, the ultimate aim is to determine the homotopy type of these path spaces.

Higher Dimensional Automata are prototypes of directed topological spaces; see Grandis [12;13]. General topological properties of spaces of d-paths and of traces (=d-paths up to monotone reparametrizations; see Fahrenberg and Raussen [5; 16]) in pre-cubical complexes were investigated in Raussen [17]. Raussen [18; 19] describes an algorithmic method to determine the homotopy types of trace spaces for Higher Dimensional Automata (and thus in particular to calculate and describe their components) through explicitly constructed finite simplicial complexes for a restricted class of model spaces:

(1) We had to stick to semaphore - or PV - models as described by Dijkstra [4] an important but restricted class of HDA. Loosely speaking, a PV-model space is a hypercube $I^{n}$ - with $I$ the unit interval $[0,1]$ - from which a number of $n$-dimensional hyperrectangles has been removed; see Raussen [18].

(2) We only considered model spaces without nontrivial directed loops.

For these restricted class of models, the resulting algorithm has meanwhile been implemented with encouraging results; see Fajstrup et al [8].

In the present paper, we propose an algorithm extending the framework to full generality yielding (generalized simplicial) models for spaces of traces in general pre-cubical complexes; hence we cover models for general (unlabeled) HDA. For these, the homotopy type of trace spaces between given end points is identified with an explicitly constructed complex (a generalization of a simplicial complex); all components of that complex are finite. Using this complex, topological invariants (eg homology) can be calculated.

A price has to be paid: the algorithm determining this complex is, at least in general, more intricate than in the semaphore case. Data structures can be much more complicated, and we have no experience with running times yet.

\subsection{Structure and overview of results}

Section 2 introduces pre-cubical complexes as HDA; we abstract away from labels. We introduce a signed $L_{1}$-arc length on general paths in a pre-cubical complex with 
positive or negative values extending the definition from Raussen [17] for d-paths. It is shown that this signed $L_{1}$-arc length is invariant under homotopy with fixed end points for all paths and that the range of the $L_{1}$-arc length map is discrete given a pair of end points.

We introduce the class of nonbranching (and nonlooping) pre-cubical complexes in Section 3. We show, that the space of traces between two points in such a complex is always either empty or contractible.

In the central Section 4, we consider traces in pre-cubical complexes with branch points but without nontrivial directed loops. We decompose such a complex into subcomplexes without branch points and such that the associated trace spaces cover the trace space corresponding to the entire complex. This decomposition can be quite complicated in the presence of higher order branch points. The nerve of the poset category associated to this cover is homotopy equivalent to trace space. Moreover, we construct a complex (with cones of products of simplices as building blocks) homotopy equivalent to trace space and "more economical" than this nerve.

In Section 5, we show that trace spaces for a pre-cubical complex with nontrivial directed loops can be analysed through trace spaces in an associated covering space in which lifts of paths depend on their $L_{1}$-arc length - and in which (nontrivial) d-loops lift to nonloops.

In the final Section 6, we give a few hints about a possible implementation that, with a pre-cubical set as input and using an associated directed graph, allows to determine the poset category describing the associated trace space.

\section{Pre-cubical complexes and length maps}

\subsection{Directed paths and traces in a pre-cubical complex}

Properties of Higher Dimensional Automata (cf Section 1.1) are intimately related to the study of directed paths in a pre-cubical set, also called a $\square$-set; this term (cf [6]) is used in a similar way as a $\Delta$-set - as introduced in [20] - for a simplicial set without degeneracies. We use $\square_{n}$ as an abbreviation for the $n$-cube $I^{n}=[0,1]^{n}$ with the product topology.

Definition 2.1 (1) A $\square$-set or pre-cubical complex $X$ is a family of disjoint sets $\left\{X_{n} \mid n \geq 0\right\}$ with face maps $\partial_{i}^{k}: X_{n} \rightarrow X_{n-1}, 1 \leq i \leq n, k=0,1$, satisfying the pre-cubical relations $\partial_{i}^{k} \partial_{j}^{l}=\partial_{j-1}^{l} \partial_{i}^{k}$ for $i<j$. 
(2) The geometric realization $|X|$ of a pre-cubical set $X$ is given as the quotient space $|X|=\left(\bigsqcup_{n} X_{n} \times \square_{n}\right) / \equiv$ under the equivalence relation induced from

$$
\left(\partial_{i}^{k}(x), t\right) \equiv\left(x, \delta_{i}^{k}(t)\right), \quad x \in X_{n+1}, \quad t=\left(t_{1}, \ldots, t_{n}\right) \in \square_{n},
$$

with

$$
\delta_{i}^{k}(t)=\left(t_{1}, \ldots, t_{i-1}, k, t_{i+1}, \ldots, t_{n}\right) .
$$

(3) A pre-cubical complex $M$ is called non-self-linked (cf [9; 17]) if, for all $n$, $x \in M_{n}$ and $0<i \leq n$, the $2^{i}\left(\begin{array}{c}n \\ i\end{array}\right)$ iterated faces

$$
\partial_{l_{1}}^{k_{1}} \cdots \partial_{l_{i}}^{k_{i}} x \in M_{n-i}, \quad k_{i}=0,1, \quad 1 \leq l_{1}<\cdots<l_{i} \leq n,
$$

are all different.

In the future, we will not distinguish between a pre-cubical complex $X$ and its geometric realization and just write $X$ for both. We will tacitly assume that all pre-cubical complexes are non-self-linked; if necessary, after a barycentric subdivision.

We are interested in directed paths in $X$. A continuous path within a cube $\square_{n}$ is a d-path, if all $n$ component functions are (not necessarily strictly) increasing. A path in $X$ is a d-path if it is the concatenation of d-paths within cubes; see Raussen [17, Definition 2.2] for details. The set of all d-paths in $X$ will be denoted $\vec{P}(X) \subset X^{I}$ with subspaces $\vec{P}(X)(\mathbf{c}, \mathbf{d})$ consisting of paths with $p(0)=\mathbf{c}$ and $p(1)=\mathbf{d}$. These spaces inherit a topology from the CO-topology on $X^{I}$ (the uniform convergence topology).

Reparametrization equivalent d-paths [5] in $X$ have the same directed image (=trace) in $X$. Dividing out the action of the monoid of (weakly increasing) reparametrizations of the parameter interval $\vec{I}$, we arrive at trace space $\vec{T}(X)(\mathbf{c}, \mathbf{d}$ ) (see Fahrenberg and Raussen [5; 16]); it is shown in Raussen [17] to be homotopy equivalent to path space $\vec{P}(X)(\mathbf{c}, \mathbf{d})$ for a far wider class of directed spaces $X$; in the latter paper, it is also shown that trace spaces enjoy nice properties; eg, they are metrizable, locally compact, locally contractible, and they have the homotopy type of a CW-complex.

Notation Within $X$ and for $x \in X$, we let $\downarrow x:=\{y \in X \mid \vec{P}(X)(y, x) \neq \varnothing\}$ denote the past of $x$.

\subsection{Length maps}

The $L_{1}$-arc length of a d-path in a pre-cubical complex was introduced and studied in Raussen [17]. The definition and important properties can be extended to general nondirected paths; for these the (signed) $L_{1}$-arc length may be negative. This goes roughly as follows: 
The signed $L_{1}$-length $l_{1}^{ \pm}(p)$ of a path $p: I \rightarrow \square_{n}$ within a cube $\square_{n}$ is defined as $l_{1}^{ \pm}(p)=\sum_{j=1}^{n} p_{j}(1)-p_{j}(0)$. For any path $p$, that is the concatenation of finitely many paths each of which is contained in a single cube, the signed $L_{1}$-length is defined as the sum of the lengths of the pieces; the result is independent of the choice of decomposition - and of the parametrization! Moreover, it is nonnegative for every d-path and positive for every nonconstant d-path.

This construction can be phrased more elegantly using differential one-forms on a cubical complex (a special case of the PL differential forms introduced by D Sullivan [21] in his approach to rational homotopy theory, or of the closed one-forms on topological spaces by Farber $[10 ; 11])$ : On an $n$-cube $e \simeq \square_{n}$, consider the particular 1 -form $\omega_{e}=d x_{1}+\cdots+d x_{n} \in \Omega^{1}\left(\square_{n}\right)$. It is obvious that $\omega_{\partial_{i}^{k}}^{k}=\left(i_{i}^{k}\right)^{*} \omega_{e}$ with $i_{i}^{k}:\left|\partial_{i}^{k} e\right| \hookrightarrow|e|$ denoting inclusion. Pasting together, one arrives at a particular (closed!) 1 -form $\omega_{X}$ on every pre-cubical set $X$ - the one-form that reduces to $\omega_{e}$ on every cell $e$ in $X$.

The signed length of a (piecewise differentiable) path $\gamma$ on $X$ can then be defined as $l_{1}^{ \pm}(\gamma)=\int_{0}^{1} \gamma^{*} \omega_{X}$ and extended to continuous paths using uniformly converging sequences of such piecewise differentiable paths. This length is invariant under orientation preserving reparametrization; it changes sign under orientation reversing reparametrization; it is additive under concatenation and nonnegative for d-paths. It yields a continuous map $l_{1}^{ \pm}: P(X)\left(x_{0}, x_{1}\right) \rightarrow \mathbf{R}$. An application of Stokes' theorem shows:

Proposition 2.2 Two paths $p_{0}, p_{1} \in P(X)\left(x_{0}, x_{1}\right)$ that are homotopic rel end points have the same signed length: $l_{1}^{ \pm}\left(p_{0}\right)=l_{1}^{ \pm}\left(p_{1}\right)$.

A more direct proof can be given along the lines of Raussen [17] using the continuous map $s: X \rightarrow S^{1}=\mathbf{R} / \mathbf{Z}$ given by $s\left(e ; x_{1}, \ldots, x_{n}\right)=\sum x_{i} \bmod 1$. It is clear from the construction, that $l_{1}^{ \pm}(p) \equiv s(p(1))-s(p(0)) \bmod 1$. As a consequence, $l_{1}^{ \pm}\left(P(X)\left(x_{0}, x_{1}\right)\right)$ is constant $\bmod 1$ and, in particular, a discrete subset of the reals. Hence, $l_{1}^{ \pm}$is constant on a connected component, ie, a homotopy class of paths in $P(X)\left(x_{0}, x_{1}\right)$.

Remark 2.3 As remarked in Raussen [17, Remark 2.8] it is not possible to extend nonnegative $L_{1}$-arc length continuously to nondirected paths.

\section{Trace spaces for nonbranching pre-cubical complexes}

In the following two sections, we will only consider nonlooping pre-cubical complexes. In such a complex $X$, the only directed loops are trivial, ie, constant. 
A (finite) such pre-cubical complex $X$ will be called nonbranching if it satisfies the following additional property:

(NB) Every vertex $v \in X_{0}$ is the lower corner vertex of a unique maximal cube $c_{v}$ in $X$. This maximal cube $c_{v}$ contains thus all cubes with lower corner vertex $v$ as a (possibly iterated) lower face.

On a nonbranching cubical complex, there is a privileged directed flow

$$
F^{X}: X \times \mathbf{R}_{\geq 0} \rightarrow X:
$$

Every element $x \in X$ is contained in the interior or the lower boundary of a uniquely determined maximal cube, ie, the maximal cube $c_{v}$ of its lowest vertex $v$. On the interior and the lower faces of such a cube $c_{v}$, this flow is locally given by the diagonal flow:

$$
F_{c}^{X}\left(c ;\left(x_{1}, \ldots, x_{n}\right) ; t\right)=\left(c ; x_{1}+t, \ldots, x_{n}+t\right) \quad \text { for } 0 \leq t \leq 1-\max _{1 \leq i \leq n} x_{i} .
$$

On a maximal vertex $v_{1}$ with $c=c_{v_{1}}=v_{1}$ (a deadlock), $F_{c}^{X}$ is defined to be constant in $t$ for $0 \leq t$.

On cubes, that are not lower boundaries of others, these flow lines are the gradient lines of the 1-form $\omega_{X}$ from Section 2.2; this is not true on such lower boundaries. Piecing together these local flows so that they satisfy the flow semigroup property yields a piecewise-linear (hence Lipschitz continuous) global flow all of whose flow lines are d-paths; note from the construction that this flow can only have equilibria at deadlocks.

Remark 3.1 At a branch point $v_{0}$ in a general (branching) pre-cubical complex $X$ (see Definition 4.3), it is not possible to construct such a flow. Diagonal flows on several maximal cubes do not fit together on their intersections.

Lemma 3.2 A finite nonbranching connected pre-cubical complex $X$ has a unique maximal vertex $v_{1}$.

Proof First of all, there is at least one maximal vertex. Otherwise, one would have d-paths of arbitrary length in $X$; hence $X$ - without nontrivial loops - could not be finite.

Suppose $v_{1}, v_{2}, \ldots, v_{k} \in X, k>1$, is a list of all maximal vertices. Consider the maximal vertices in the common past subcomplexes $\downarrow v_{i} \cap \downarrow v_{j}, i \neq j$, and choose among those the maximal ones (that cannot reach any of the others). Pick such a maximal vertex $v$ and consider the associated maximal cube $c_{v}$. 
There is at least one edge in $c_{v}$ with $v$ as lower boundary from which one can reach $v_{i}$ and not $v_{j}$; likewise another edge from which one can reach $v_{j}$ and not $v_{i}$. From the top edge of $c_{v}$, at least one of the $v_{r}$ in the list is reachable. As a consequence, from at least one of the two edges mentioned before, two maximal vertices can be reached. Contradiction to maximality!

The key Proposition 2.8 from Raussen [18] generalizes as follows:

Proposition 3.3 For every pair of elements $x_{0}, x_{1} \in X$ in the geometric realization $X$ of a pre-cubical nonbranching complex $X$, trace space $\vec{T}(X)\left(x_{0}, x_{1}\right)$ is either empty or contractible.

Proof We assume that $\vec{T}(X)\left(x_{0}, x_{1}\right) \neq \varnothing$ and, without restriction, that $x_{1}$ is the maximal vertex in $X$; in general, just replace $X$ by $\downarrow x_{1} \subset X$, still a nonbranching complex; without deadlocks and unsafe regions.

The directed flow line corresponding to the flow $F^{X}$ (cf (3-1)) starting at $x \in X$ and ending at $x_{1}$ (after linear renormalization so that its domain becomes the unit interval $I)$ will be called $p_{x} \in \vec{P}(X)\left(x, x_{1}\right)$.

A contraction $H: \vec{P}(X)\left(x_{0}, x_{1}\right) \times I \rightarrow \vec{P}(X)\left(x_{0}, x_{1}\right)$ to the flow path $H_{0}=p_{x_{0}}$ is constructed as follows: For $p \in \vec{P}(X)\left(x_{0}, x_{1}\right)$, let

$$
H(p, t)(s)= \begin{cases}p(s) & t \leq s, \\ p_{p(t)}\left(\frac{s-t}{1-t}\right) & s \leq t .\end{cases}
$$

Remark that $H_{1}=p$ and that an intermediate d-path $H_{t}$ follows $p$ until $p(t)$ and then it follows the flow line starting at $p(t)$ automatically ending at $x_{1}$.

Finally, the quotient map $\vec{P}(X)\left(x_{0}, x_{1}\right) \rightarrow \vec{T}(X)\left(x_{0}, x_{1}\right)$ is a homotopy equivalence [17].

This proof, using the diagonal flow $F^{X}$, is different from the one given in Raussen [18, Proposition 2.8] for the special case of cubical complexes arising from semaphore models; but it is certainly similar in spirit.

\section{Trace spaces for nonlooping pre-cubical complexes}

In this section, we study traces in a more general finite pre-cubical complex $X$; still without nontrivial loops, but allowing for branch points: How to find subcomplexes $Y \subseteq X$ satisfying (NB)? Investigating the space of d-paths between $\mathbf{x}_{0}$ and $\mathbf{x}_{1}$ in $X$, we assume that $X=\left[\mathbf{x}_{0}, \mathbf{x}_{1}\right]=\uparrow \mathbf{x}_{0} \cap \downarrow \mathbf{x}_{1}$. In particular, $X$ contains neither unsafe nor unreachable regions. We start with an abstract description: 


\subsection{An abstract simplicial model}

The subcomplex given by the carrier sequence corresponding to any directed path (see Fajstrup [6]), the sequence of cubes containing segments of that path, is obviously a subcomplex satisfying $(\mathrm{NB})$.

One may order NB subcomplexes of $X$ by inclusion - chains are of bounded length since there are only finitely many cubes - and focus on the maximal nonbranching subcomplexes. Every d-path with a given start point is contained in a maximal NB subcomplex, that is in general not uniquely determined. Traces contained in maximal NB subcomplexes cover thus the space of all d-paths (with given start and end point).

Lemma 4.1 An intersection $S=\bigcap X_{i}$ of subcomplexes $X_{i}$ each satisfying (NB) satisfies (NB) as well. Hence the trace space $\vec{T}(S)\left(\mathbf{x}_{0}, \mathbf{x}_{1}\right)$ is either contractible or empty.

Proof The intersection of maximal cubes at every vertex will be the maximal cube in the intersection and hence unique. For contractibility, use Proposition 3.3. Empty path spaces may arise when $S$ is not connected.

The subcomplexes $X_{i} \subset X, i \in I$, satisfying (NB) that are maximal with respect to inclusion give thus rise to a covering $\vec{T}\left(X_{i}\right)\left(\mathbf{x}_{0}, \mathbf{x}_{1}\right)$ of trace space $\vec{T}(X)\left(\mathbf{x}_{0}, \mathbf{x}_{1}\right)$ by contractible sets; in fact:

Theorem 4.2 For a finite pre-cubical complex $X$, the trace space $\vec{T}(X)\left(\mathbf{x}_{0}, \mathbf{x}_{1}\right)$ is homotopy equivalent to the nerve of the covering given by the subspaces $\vec{T}\left(X_{i}\right)\left(\mathbf{x}_{0}, \mathbf{x}_{1}\right)$.

Proof The theorem is an almost immediate consequence of the nerve lemma; see Kozlov [14, Theorem 15.21]. The subspaces $X_{i}$ are in general not open, and the subspaces $\vec{T}\left(X_{i}\right)\left(\mathbf{x}_{0}, \mathbf{x}_{1}\right)$ will in general not give rise to an open covering. According to Raussen [17, Proposition 3.15], the trace space $\vec{T}(X)\left(\mathbf{x}_{0}, \mathbf{x}_{1}\right)$ has the homotopy type of a CW-complex; the subspaces $\vec{T}\left(X_{i}\right)\left(\mathbf{x}_{0}, \mathbf{x}_{1}\right)$ correspond to subcomplexes. The projection lemma [14, Theorem 15.19 and Remark 15.20] comparing homotopy colimits with colimits holds also in this case.

\subsection{An index category}

4.2.1 (Higher order) branch points The maximal subcomplexes $X_{i}$ from Theorem 4.2 may be very difficult to identify for a complex $X$ with many cells. In the following, we describe an algorithmic method that determines an index category $\mathcal{C}(X)\left(\mathbf{x}_{0}, \mathbf{x}_{1}\right)$ 
that can be represented by a complex $\mathbf{T}(X)\left(\mathbf{x}_{0}, \mathbf{x}_{1}\right)$ which is homotopy equivalent to trace space $\vec{T}(X)\left(\mathbf{x}_{0}, \mathbf{x}_{1}\right)$. The building blocks of the complex $\mathbf{T}(X)\left(\mathbf{x}_{0}, \mathbf{x}_{1}\right)$ are products of simplices and of cones of such spaces. The construction is similar in spirit to that in Raussen [18], albeit, in the details slightly more complicated.

We investigate vertices in the 0 -skeleton $X_{0} \subset X$ at which condition (NB) from Section 4 are violated:

Definition 4.3 A vertex $v \in X_{0}$ is called a branch point if there are more than one maximal cube $c$ having $v$ as lower vertex (ie, an iterate of $\partial_{*}^{0}$ yields $v$ ). The set of all such maximal cells with lower vertex $v$ is called the branch set $B_{v}$ with $\left|B_{v}\right|>1$.

Remark 4.4 Let $v \in X_{0}$ be a branch point with several maximal cubes $c_{1}, \ldots, c_{r}$ with lower vertex $v$. Obviously, at most one of the cubes $c_{j}$ can be contained in every of the (NB) subcomplexes $X_{i}$ from Section 4.1.

For a cell $c$ in $X$, we denote by $c^{-}$the geometric realization of $c$ and of all (iterated) lower boundaries - not including mixed or upper boundaries. Hence, $\left|c^{-}\right| \cong\left[0,1\left[^{n}\right.\right.$ for an $n$-cell $c$. For a fixed branch point $v$ and a branch cell $c_{j} \in B_{v}$, let

$$
X_{j}^{v}:=\downarrow c_{j}^{-} \cup \mathcal{C}\left(\downarrow \bigcup_{c_{i} \in B_{v}} c_{i}^{-}\right)
$$

consist of all points that, as far as they can reach any branch in $B_{v}$, they have to stay in the past of the particular branch $c_{j} ; \mathcal{C}$ denotes the complement within $X$. Clearly, $X=\bigcup_{c_{j} \in B_{v}} X_{j}^{v}$.

Lemma 4.5 $\vec{T}(X)\left(\mathbf{x}_{0}, \mathbf{x}_{1}\right)=\bigcup_{c_{j} \in B_{v}} \vec{T}\left(X_{j}^{v}\right)\left(\mathbf{x}_{0}, \mathbf{x}_{1}\right)$ for every branch point $v$.

Proof We need to show that $\vec{T}\left(\bigcup_{c_{j} \in B_{v}} X_{j}^{v}\right)\left(\mathbf{x}_{0}, \mathbf{x}_{1}\right)=\bigcup_{c_{j} \in B_{v}} \vec{T}\left(X_{j}^{v}\right)\left(\mathbf{x}_{0}, \mathbf{x}_{1}\right):$ Every d-path from $\mathbf{x}_{0}$ to $\mathbf{x}_{1}$ starts in the (past closed) set $\downarrow \bigcup_{c_{j} \in B_{v}} c_{j}^{-}$and then leaves it for its (future closed) complement. The sets $\downarrow c_{j}^{-}$are all past closed; a d-path $p$ that has left one of these sets will never get back to it. In particular, there is at least one (last) set $X_{j}^{v}, c_{j} \in B_{v}$, containing $p$.

Contrary to the special situation of pre-cubical complexes arising from $P V$-protocols discussed in [18], it is not enough to consider only (1. order) branch points as the following example (cf Figure 1) shows: 


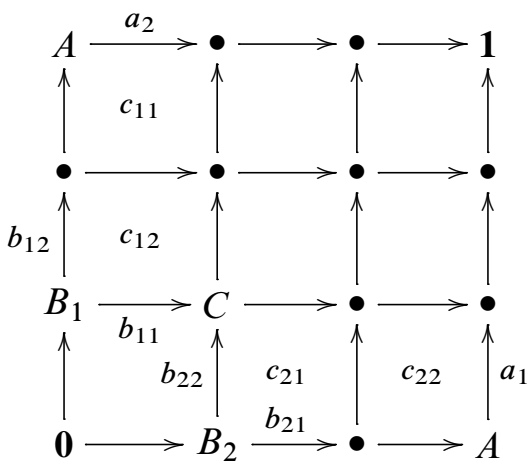

Figure 1: Branch points in a 2D complex

Example 4.6 The complex $X$ to be discussed arises from the 9 planar 2-cubes in Figure 1 by identifying the two vertices denoted $A$. Remark the two special "horizontal" and "vertical" d-paths from $\mathbf{0}$ to $\mathbf{1}$ through $A$. The vertex $A$ is the only branch point in $X$; it has branch set $B^{A}=\left\{a_{1}, a_{2}\right\}$. The subcomplex $X_{1}^{A}$ arises from $X$ by crossing out the two cells $c_{11}$ and $c_{12}$ - apart from the left boundary 1-cells. Likewise, for $X_{2}^{A}$, the cells $c_{21}$ and $c_{32}$ - apart from the lower boundary 1-cells - have to be deleted. The first subcomplex has a secondary branch point $B_{1}$ with branches $b_{11}$ and $b_{12}$. Likewise, the second one has a secondary branch point $B_{2}$ with branches $b_{21}$ and $b_{22}$.

The homotopy type of the trace space $\mathbf{T}(X)(\mathbf{0}, \mathbf{1})$ will be identified in Example 4.12 below.

4.2.2 The index category $\mathcal{C}(X)\left(\mathbf{x}_{0}, \mathbf{x}_{1}\right)$ Hence, it is necessary to consider secondary, and in general higher order branch points, as well:

- The original space $X$ comes with a set $\mathrm{BP}=\left\{b^{1}, \ldots, b^{l}\right\}$ of branch points and associated maximal branch cubes $\mathrm{BC}=\left\{c_{j}^{i}\right\}$ and a surjective map $p$ : $\mathrm{BC} \downarrow \mathrm{BP}$, $p\left(c_{j}^{i}\right)=b^{i}$.

- For every section $s(1)$ : $\mathrm{BP} \uparrow \mathrm{BC}$ of the map $p$, consider the subcomplex $X_{s(1)}=$ $\bigcap_{b_{i} \in \mathrm{BP}}\left(\downarrow c_{s(1)\left(b_{i}\right)}^{i-} \cup \mathcal{C}\left(\bigcup_{p(j)=b_{i}} \downarrow c_{j}^{i-}\right) \subset X . X_{s(1)}\right.$ is in fact a subcomplex of $X$ since the branch cubes are all maximal. It is a proper subcomplex containing $\mathbf{x}_{0}$ and $\mathbf{x}_{1}$.

- Such a complex $X_{s(1)}$ may have (second order) branch points $b^{i}(s(1)) \in$ $\mathrm{BP}_{s(1)}$ and branch cubes $c_{j}^{i}(s(1)) \in \mathrm{BC}_{s(1)}$ which come with a projection $p(s(1)): \mathrm{BC}_{s(1)} \downarrow \mathrm{BP}_{s(1)}$ and sections $s(2): \mathrm{BP}_{s(1)} \uparrow \mathrm{BC}_{s(1)}$. 
- Iterate: Given subsequent sections $s(1), \ldots, s(r)$ define a proper subcomplex $X_{s(r)} \subset X_{s(r-1)}$ as

$$
X_{s(r)}=\bigcap_{0 \leq k \leq r} \bigcap_{b_{i} \in \mathrm{BP}(s(k))}\left(\downarrow c_{s(k)\left(b_{i}\right)}^{i-} \cup \mathcal{C}\left(\bigcup_{p(k)(j)=b_{i}} \downarrow c_{j}^{i-} ; X_{s(k-1)}\right)\right),
$$

with the convention that $p(s(0))$ : $\mathrm{BC}(s(0)) \downarrow \mathrm{BP}(s(0))$ is the original projection map $p$ : $\mathrm{BC} \downarrow \mathrm{BP}$. This complex $X_{s(r)}$ may give rise to sets of new branch cells $\mathrm{BC}_{s(r)}$ and branch points $\mathrm{BP}_{s(r)}$ with a projection map $p(s(r))$ : $\mathrm{BC}_{s(r)} \downarrow \mathrm{BP}_{s(r)}$. Since these subcomplexes become smaller and smaller under iteration in the finite complex $X$, every such iteration will ultimately end in a subcomplex $X_{S(r)}$ without branch points.

- A subsequent sequence of sections $s(k): \mathrm{BP}_{s(k)} \uparrow \mathrm{BC}_{s(k)}, k \leq r$, is called coherent and complete if $X_{s(r)}$ satisfies property (NB); cf Section 3. The set of all such coherent and complete sequences will be called $\operatorname{CCS}(X)$.

- Given a coherent and complete sequence $s \in \operatorname{CCS}(X)$, we may associate the set of branch points $\mathrm{BP}(s)=\bigcup_{k=0}^{r} \mathrm{BP}_{s(k)}$ and branch cubes $\mathrm{BC}(s)=$ $\bigcup_{k=0}^{r} \mathrm{BC}_{s(k)}$, the projection $p(s)$ : $\mathrm{BC}(s) \downarrow \mathrm{BP}(s)$, and the "tautological" section $\bar{s}: \mathrm{BP}(s) \uparrow \mathrm{BC}(s)$; there are no branch cells to choose at depth $s$ !

\section{Definition 4.7 (1) The poset category $\mathcal{M}(X)\left(\mathbf{x}_{0}, \mathbf{x}_{1}\right)$ has as}

- objects all pairs of the form $(S, C)$ with $\varnothing \neq S \subset \operatorname{CCS}(X)$ and $C$ a set of the form $C=\prod_{b_{i} \in \cup_{s \in S} \mathrm{BP}(s)} C_{i}, \bar{s}\left(b_{i}\right) \in C_{i} \subset \mathrm{BC}\left(b_{i}\right)$; with $\bar{s}$ denoting the tautological section from Section 4.2.2,

- morphisms $(S, C) \leq\left(S^{\prime}, C^{\prime}\right) \Leftrightarrow S \subseteq S^{\prime}, \forall s \in S, b_{i} \in \mathrm{BP}(s): C_{i} \subseteq C_{i}^{\prime}$.

Note that the minimal objects $(S, D)$ of this category are composed of a set $S$ with precisely one element $s$ and such that $b_{i} \in \mathrm{BP}(s) \Rightarrow\left|C_{i}\right|=1$.

(2) To a section $s \in \operatorname{CCS}(X)$, a branch point $b_{i} \in \operatorname{BP}(s(k))$ and a branch cube $c_{i} \in \mathrm{BC}(s(k))$, we associate the subspace $\left(X_{s(k)}\right)_{b_{i}}^{c_{i}} \subset X$. To an object $(S, C)$ in $\mathcal{M}(X)\left(\mathbf{x}_{0}, \mathbf{x}_{1}\right)$ we associate the subspace $X_{(S, C)}:=I \subset X$.

(3) The category $\mathcal{C}(X)\left(\mathbf{x}_{0}, \mathbf{x}_{1}\right)$ is the full subcategory of $\mathcal{M}(X)\left(\mathbf{x}_{0}, \mathbf{x}_{1}\right)$ whose objects $(S, C)$ are characterized by the fact that $\vec{T}\left(X_{(S, C)}\right)\left(\mathbf{x}_{0}, \mathbf{x}_{1}\right)$ is nonempty.

Proposition 4.8 (1) $\vec{T}(X)\left(\mathbf{x}_{0}, \mathbf{x}_{1}\right)=\bigcup_{(S, C)} \vec{T}\left(X_{(S, C)}\right)\left(\mathbf{x}_{0}, \mathbf{x}_{1}\right)$, where the union extends over all objects of the category $\mathcal{C}(X)\left(\mathbf{x}_{0}, \mathbf{x}_{1}\right)$.

(2) The subspace $\vec{T}\left(X_{(S, C)}\right)\left(\mathbf{x}_{0}, \mathbf{x}_{1}\right)$ is contractible for every object $(S, C)$ of the subcategory $\mathcal{C}(X)\left(\mathbf{x}_{0}, \mathbf{x}_{1}\right)$. 
Proof (1) This follows from Lemma 4.5 by induction.

(2) According to Proposition 3.3, the trace space $\vec{T}\left(X_{s(r)}\right)\left(\mathbf{x}_{0}, \mathbf{x}_{1}\right)$ is empty or contractible for every complete coherent sequence of branches and branch points. For every object $(S, C)$ of the category $\mathcal{C}(X)\left(\mathbf{x}_{0}, \mathbf{x}_{1}\right)$, the space $X_{(S, C)}$ is a finite intersection of spaces of type $\vec{T}\left(X_{s(r)}\right)\left(\mathbf{x}_{0}, \mathbf{x}_{1}\right), s \in S$. Apply Lemma 4.1 .

Remark 4.9 An algebraic representation of the category $\mathcal{C}(X)\left(\mathbf{x}_{0}, \mathbf{x}_{1}\right)$ is not as straightforward as for semaphore models that resulted in a poset category of binary matrices (cf Raussen [18]). In the general case, one has to consider combinations of all branch point sequences (of different lengths) and the occuring branches - which may have different cardinalities. I plan to develop algorithmically the representation of $\mathcal{C}(X)\left(\mathbf{x}_{0}, \mathbf{x}_{1}\right)$ in a future paper.

\subsection{Homotopy equivalences}

We will now present a description of the homotopy type of $\vec{T}(X)\left(\mathbf{x}_{0}, \mathbf{x}_{1}\right)$ as a colimit of simple spaces (generalizing the prodsimplicial complex for traces arising from semaphore models; cf Raussen [18]). For simplicity, we will describe only the case with branch points of order at most two.

In this case, to every first order branch point $b^{i} \in \mathrm{BP}$, we associate the branch cells $\mathrm{BC}^{i}=p^{-1}\left(b^{i}\right)$; a section $s$ : $\mathrm{BP} \uparrow \mathrm{BC}$ gives rise to second order branch points in $\mathrm{BP}(s)$ and, to every of the second order branch points $b^{i}(s) \in \mathrm{BP}(s)$, a set of branch cells $\mathrm{BC}^{i}(s)$. Then, the total complex corresponding to all objects $(S, C)$ corresponds to

$$
\Delta^{\left|\mathrm{BC}^{1}\right|-1} \times \cdots \times \Delta^{\left|\mathrm{BC}^{l}\right|-1} \times \prod_{s: \mathrm{BP} \uparrow \mathrm{BC}} C\left(\Delta^{\left|\mathrm{BC}^{1}(s)\right|-1} \times \cdots \times \Delta^{\left|\mathrm{BC}^{l(s)}(s)\right|-1}\right) .
$$

Here $C X$ denotes the cone over $X$.

A subset $S=S_{1} \times \cdots \times S_{l} \subseteq \mathrm{BC}^{1} \times \cdots \times \mathrm{BC}^{l}$ corresponds to a product of simplices $\Delta_{S}=\Delta^{\left|S_{l}\right|-1} \times \cdots \times \Delta^{\left|S_{1}\right|-1}$. A subset $C(s)=\prod_{b^{i}(s) \in \mathrm{BP}(s)} C^{i}(s), s: \mathrm{BP} \uparrow \mathrm{BC}$, of products of first and second order branch cells corresponds to

$$
\Delta^{C}(s):=\Delta^{\left|C^{1}(s)\right|-1} \times \cdots \times \Delta^{\left|C^{l(s)}(s)\right|-1} \subseteq \Delta^{\left|\mathrm{BC}^{1}(s)\right|-1} \times \cdots \times \Delta^{\left|\mathrm{BC}^{l(s)}(s)\right|-1} .
$$

An object $(S, C)$ in $\mathcal{M}(X)\left(\mathbf{x}_{0}, \mathbf{x}_{1}\right)$ corresponds to

$$
\Delta(S, C):=\Delta_{S} \times \prod_{s \in S} C\left(\Delta^{C}(s)\right) \times \prod_{s \notin S} *_{s},
$$

with $*_{s}$ the cone point in $C\left(\Delta^{C}(s)\right)$. Morphisms $(S, C) \leq\left(S^{\prime}, C^{\prime}\right)$ correspond to inclusions $\Delta(S, C) \hookrightarrow \Delta\left(S^{\prime}, C^{\prime}\right)$. 
Definition 4.10 The complex $\mathbf{T}(X)\left(\mathbf{x}_{0}, \mathbf{x}_{1}\right)$ is defined as the colimit

$$
\mathbf{T}(X)\left(\mathbf{x}_{0}, \mathbf{x}_{1}\right):=\operatorname{colim}_{\mathcal{C}(X)\left(\mathbf{x}_{0}, \mathbf{x}_{1}\right)} \Delta(S, C) .
$$

Theorem 4.11 The trace space $\vec{T}(X)\left(\mathbf{x}_{0}, \mathbf{x}_{1}\right)$ is homotopy equivalent to

(1) the nerve $\Delta\left(\mathcal{C}(X)\left(\mathbf{x}_{0}, \mathbf{x}_{1}\right)\right)$ of the poset category $\mathcal{C}(X)\left(\mathbf{x}_{0}, \mathbf{x}_{1}\right)$, and

(2) the complex $\mathbf{T}(X)\left(\mathbf{x}_{0}, \mathbf{x}_{1}\right)$.

Proof The proof of Theorem 4.11 is analogous to that of [18, Theorem 3.5]: The homotopy colimit of the functor associating the contractible spaces $\vec{T}(X)_{(S, C)}\left(\mathbf{x}_{0}, \mathbf{x}_{1}\right)$, resp. $\Delta(S, C)$ to an object $(S, C)$ in $\mathcal{C}(X)\left(\mathbf{x}_{0}, \mathbf{x}_{1}\right)$ is homotopy equivalent to the functor associating the same point to every $(S, C)$, ie to the nerve of that category. Homotopy colimit and colimit of the first two functors are also homotopy equivalent; by Lemma 4.5 , this colimit is the entire trace space, resp. by definition the complex $\mathbf{T}(X)\left(\mathbf{x}_{0}, \mathbf{x}_{1}\right)$.

\subsection{Example: Trace spaces for 2-dimensional pre-cubical complexes}

Example 4.12 First, we look at the case of the space $X$ described in Example 4.6 and Figure 1. There are four coherent and complete sequences of sections:

$$
\begin{array}{ll}
s_{1}(A)=a_{1}, & s_{1}(1)\left(B_{1}\right)=b_{11}, \\
s_{2}(A)=a_{1}, & s_{2}(1)\left(B_{1}\right)=b_{12}, \\
s_{3}(A)=a_{2}, & s_{3}(1)\left(B_{2}\right)=b_{21}, \\
s_{4}(A)=a_{2}, & s_{4}(1)\left(B_{2}\right)=b_{22},
\end{array}
$$

corresponding to minimal objects $\left(S_{i}=\left\{s_{i}\right\}, C_{i}\right)$ with $C_{i}$ the one-element set given by the branches chosen by the section.

The only nontrivial intersection occurs for

$$
\vec{T}\left(X_{A}^{a_{1}} \cap X_{B_{1}}^{b_{11}}\right)(\mathbf{0 , 1}) \quad \text { and } \quad \vec{T}\left(X_{A}^{a_{2}} \cap X_{B_{2}}^{b_{22}}\right)(\mathbf{0}, \mathbf{1})
$$

giving rise to the object $\left(S_{14}=\left\{s_{1}, s_{4}\right\}, C_{14}=\left\{a_{1}, a_{2}\right\} \times\left\{b_{11}\right\} \times\left\{b_{22}\right\}\right)$. In this case, the complex $\mathbf{T}(X)(\mathbf{0}, \mathbf{1})$ is thus a disjoint union of the cones on two vertices (each of this edges corresponds to one of the special horizontal and vertical traces) and product of an edge with the cone on a vertex.

Using Theorem 4.11, we can conclude: $\vec{T}(X)(\mathbf{0}, \mathbf{1}) \simeq \mathbf{T}(X)(\mathbf{0}, \mathbf{1}) \cong I \sqcup I \sqcup I^{2}$ is homotopy equivalent to a set of three disjoint points. 
Example 4.13 The 2-dimensional complex $X$ in Figure 2 below arises from gluing the boundaries $\partial \square^{3}$ of two 3-cubes $\square^{3}$ along a common face $\square^{2}$. Its trace space has previously been studied by Bubenik [3]. The complex has two branch points $\mathbf{x}_{0}$ and $A$ and no higher order branch points.

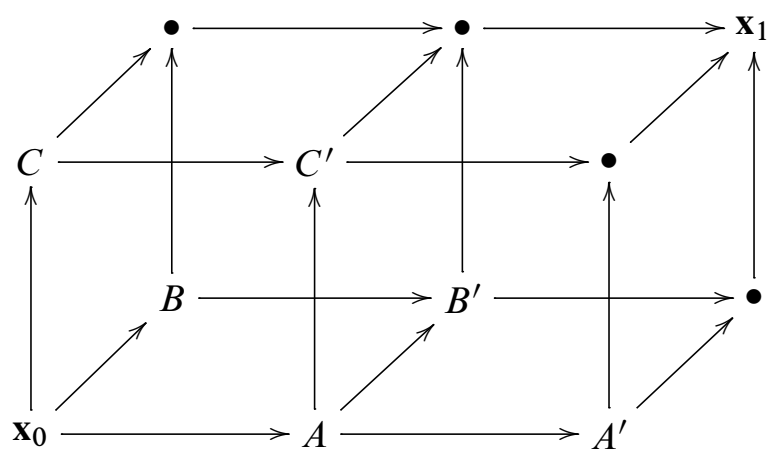

Figure 2: The complex $X$ : Boundaries of two cubes glued together at common square $A B^{\prime} C^{\prime} \bullet$

Bubenik's "necklace" model yields a simplicial complex consisting of 16 triangles, 46 edges and 29 vertices which is seen to be homotopy equivalent to $S^{1} \vee S^{1}$. The complex $\mathbf{T}(X)\left(\mathbf{x}_{0}, \mathbf{x}_{1}\right)$ - a prodsimplicial complex in the terminology of Kozlov [14], since there are no higher order branch points - homotopy equivalent to $\vec{T}(X)\left(\mathbf{x}_{0}, \mathbf{x}_{1}\right)$ (cf Theorem 4.11 above) is shown in Figure 3.
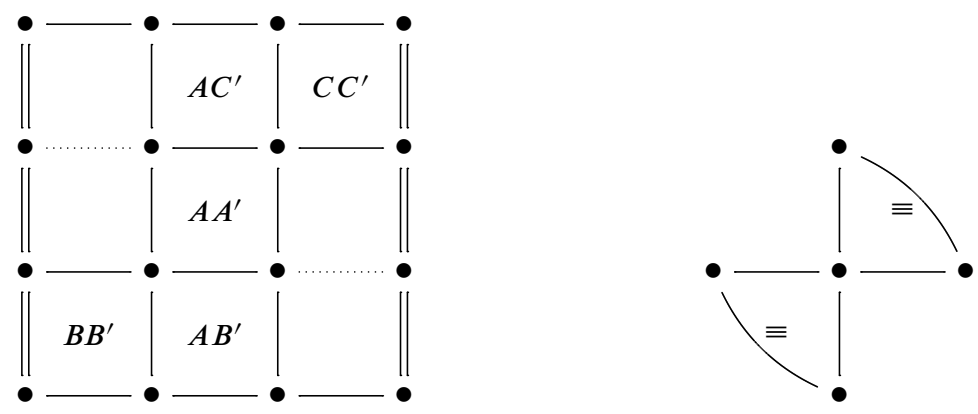

Figure 3: Prodsimplicial complex homotopy equivalent to the trace space for $X-$ and a homotopy equivalent complex

It consists of the five named squares in the nine square decomposition of a 2-torus $\Delta^{1} \times \Delta^{1}$ - identify boundary edges as usual - to which a full triangle has been attached 
along the circle on the vertical (left=right) triangle (marked by double lines $=$ ). The nine vertices correspond to $3 \times 3$ combinations of paths staying "under" the three branches (2-cubes) corresponding to the two branch points $x_{0}$ and $A$ in Figure 2.

The labels in the five marked squares refer to paths staying under two branches for each of the branch points. They are marked by labels referring to traces through the points mentioned. For example, $A C^{\prime}$ denotes the space of all traces from $\mathbf{x}_{0}$ to $A$ (front and bottom of the first cube) and then $C^{\prime}$ (front and left of the second cube) and from $C^{\prime}$ as an arbitrary d-path on the top square of the second cube to $\mathbf{x}_{1}$. It is easily seen, that there are no d-paths corresponding to the remaining four squares $\mathrm{BC}^{\prime}, B A^{\prime}, C A^{\prime}$ and $C B^{\prime}$.

Furthermore, there is a full triangle (marked with $=$ ): Every trace entering the interior of the "left" square $x_{0} B \bullet C$ in Figure 2 leaves the past of the union of the branches corresponding to branch point $A$; such a path is therefore contained in all three sets $X_{j}^{A}$, giving rise to a full triangle $\Delta^{0} \times \Delta^{2}$.

The complex $\mathbf{T}(X)\left(x_{0}, x_{1}\right)$ in Figure 3 consists thus of six 2-cells (five of type $\Delta^{1} \times \Delta^{1}$, one of type $\Delta^{0} \times \Delta^{2}$ ), 16 edges (all but the two stipled ones) and of all 9 vertices; it has Euler characteristic -1 . A contraction of the full triangle can be extended to a contraction of the entire space to a union of two full triangles (shown on the right hand side of Figure 3) with three vertices (opposite vertices are identified). That simplicial 2-complex contracts to a 1-complex $S^{1} \vee S^{1}$.

From Theorem 4.11, we may conclude: $\vec{T}(X)\left(\mathbf{x}_{0}, \mathbf{x}_{1}\right) \simeq S^{1} \vee S^{1}$.

\section{Trace spaces for general pre-cubical complexes}

In this section we outline, how the methods previously explained can be adapted to trace spaces in a general pre-cubical complex $X$ that may allow directed loops using suitable coverings of the complex $X$ :

\subsection{Nonlooping length coverings}

We exploit the d-map (directed map) $s: X \rightarrow \vec{S}^{1} \cong \mathbf{R}_{/ \mathbf{Z}}$ introduced in Raussen [17]: just glue the maps $s\left(x_{1}, \ldots, x_{n}\right)=\sum x_{i} \bmod 1$ on individual cubes. Consider the pullback $\tilde{X}$ in the pullback diagram

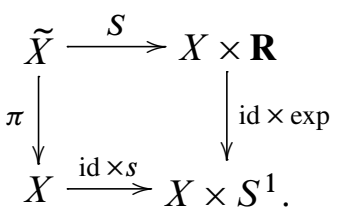


The map $\pi$ is a covering map with unique path lifting. Since exp can be interpreted as a semi-cubical map, $\widetilde{X}$ can be conceived as a semi-cubical complex: every cube $e$ in $X$ is replaced by infinitely many cubes $(e, n), n \in \mathbf{Z}$ with boundary maps given as $\partial_{-}(e, n)=\left(\partial_{-} e, n\right), \partial_{+}(e, n)=\left(\partial_{+} e, n+1\right)$.

The directed paths on $\tilde{X}$ are those that project to directed paths in $X$ under the projection map $\pi$. Note the maps exp and $s$-and hence $\pi$ and $\pi_{2} \circ S$-preserve the signed $L_{1}$-arc lengths from Section 2.2. Moreover, the $L_{1}$-length $l_{1}^{ \pm}(\underset{\widetilde{X}}{p})$ of a path $p$ in $X$ (cf Section 2.2) with lift $\tilde{p}$ can be expressed via the d-map $S: \widetilde{X} \rightarrow X \times \mathbf{R}$ in the pullback diagram as follows:

\section{Lemma $5.1 \quad(1) \quad l_{1}^{ \pm}(p)=\pi_{2}(S(\tilde{p}(1)))-\pi_{2}(S(\tilde{p}(0)))$.}

(2) $\tilde{X}$ has only trivial directed loops.

Proof (1) This is clearly true locally in any cell as long as start and end point have an $L_{1}$-distance less than one. Sum up and cancel!

(2) A directed path in $\tilde{X}$ projects to a directed path in $X$ with positive $L_{1}$-length, unless it is constant. Apply (1).

Another method to construct this covering is to consider the homotopical length map

$$
\pi_{1}(X) \stackrel{l_{1}^{ \pm}}{\rightarrow} \mathbf{Z} \rightarrow 0
$$

(cf Proposition 2.2) from the nondirected classical fundamental group of the cubical complex $X$. Consider the cover $\tilde{X} \downarrow X$ with fundamental group $\pi_{1}(\tilde{X})=K \unlhd \pi_{1}(X)$ the kernel of the homotopical length map. It can be given the structure of a pre-cubical complex, and every element $\mathbf{x}$ in $X$ has lifts $\mathbf{x}^{n} \in \tilde{X}, n \in \mathbf{Z}$. The projection map $\pi: \tilde{X} \downarrow X$ preserves signed $L_{1}$-arc length. A path in $\tilde{X}$ is directed if its projection to $X$ is. There are no nontrivial directed loops in $\tilde{X}$ - these need to have $L_{1}$-length 0 !

Example 5.2 (1) Consider a torus $T=\partial \Delta^{1} \times \partial \Delta^{1}$ as a pre-cubical set consisting of nine 2 -cubes. The length cover $\tilde{T}$ can then be modeled as an infinite strip of width 3 with identifications $(x, 3) \sim(x+3,0)$; see Figure 4 .

The subcomplex in Figure 4 between an initial vertex $\mathbf{0}$ and a second final vertex $\mathbf{1}$ at length distance $3 n, n>0$, has exactly one branch vertex $\bullet$ ( 3 to the left $\sim$ below the final vertex). The algorithm deriving the homotopy type of all d-paths of length $3 n$ between these two vertices (consisting of $n+1$ contractible components corresponding to pairs $(k, l)$ of nonnegative integers with $k+l=n)$ runs through many higher order branch points and removes only few cells at a time. 


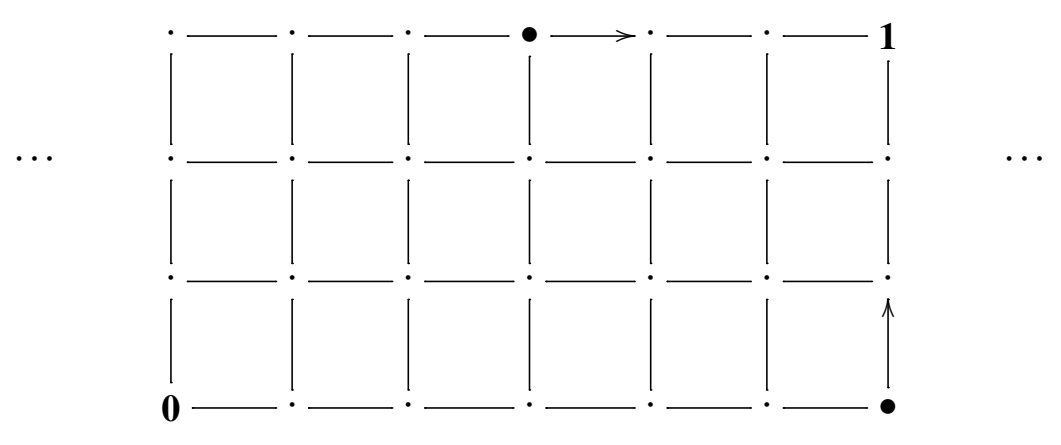

Figure 4: Length cover of a torus

(2) Now consider the space $\bar{T}$ arising from removing a (middle) cell in $T$. The length cover of $\bar{T}$ arises from $\widetilde{T}$ by removing every third cell (marked $X$ ) in the middle strip; cf Figure 5. The lower corner vertices of the removed cells are all branch vertices.

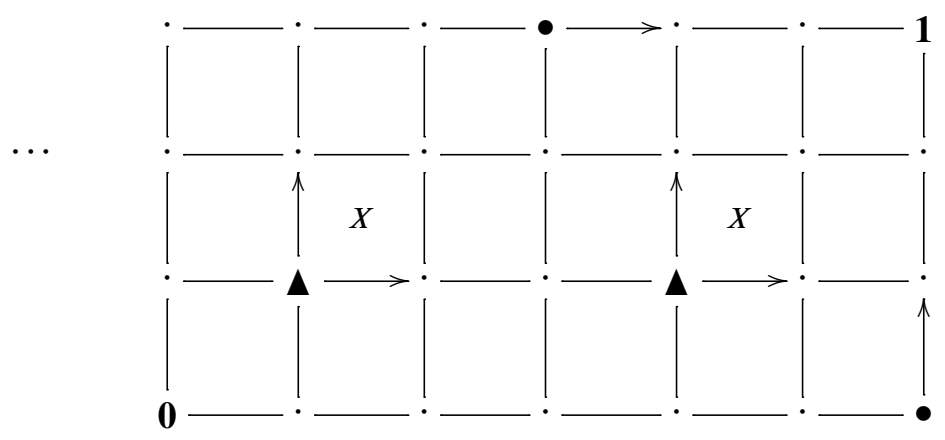

Figure 5: Length cover of torus with removed cell

For this space, no higher order branch points arise; the $2^{n}$ contractible components of the trace space correspond to the sequences of length $n$ on the letters $r, u$-right and up.

\subsection{A decomposition of the trace space}

For a general pre-cubical complex $X$ with length cover $\tilde{X}$ we obtain:

Proposition 5.3 For every pair of points $\mathbf{x}_{0}, \mathbf{x}_{1} \in X$, trace space $\vec{T}(X)\left(\mathbf{x}_{0}, \mathbf{x}_{1}\right)$ is homeomorphic to the disjoint union $\bigsqcup_{n \in \mathbf{Z}} \vec{T}(\tilde{X})\left(\mathbf{x}_{0}^{0}, \mathbf{x}_{1}^{n}\right)$. 
Proof An inverse to the projection map $\Pi: \bigsqcup_{n \in \mathbf{Z}} \vec{T}(\tilde{X})\left(\mathbf{x}_{0}^{0}, \mathbf{x}_{1}^{n}\right) \rightarrow \vec{T}(X)\left(\mathbf{x}_{0}, \mathbf{x}_{1}\right)$ induced by the covering projection $\pi: \tilde{X} \downarrow X$ is given by unique lifts of the directed paths representing traces. Remark that many of the spaces $\vec{T}(\tilde{X})\left(\mathbf{x}_{0}^{0}, \mathbf{x}_{1}^{n}\right)$ may be empty for specific $n \in \mathbf{Z}$.

Since the covering $\tilde{X}$ has only trivial loops, Proposition 5.3 allows us to apply the methods from Section 4 in order to describe the homotopy type of trace spaces $\vec{T}(X)\left(\mathbf{x}_{0}, \mathbf{x}_{1}\right)$ in an arbitrary pre-cubical complex $X$. It is of course desirable to exploit periodicity properties in the comparison of spaces $\vec{T}(\widetilde{X})\left(\mathbf{x}_{0}^{0}, \mathbf{x}_{1}^{n}\right)$ for different values of $n$.

Remark 5.4 Simple semaphore models with loops can be constructed from spaces of the form $X=T^{n} \backslash F$ with $T^{n}=\left(S^{1}\right)^{n}$ an $n$-torus and $F$ a collection of forbidden hyperrectangles. For such a space, one may consider the (sub)covering

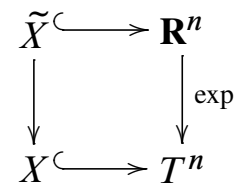

of the universal covering of $X-\mathrm{a}$ far bigger gadget. It has the property that (d-)paths, that are not homotopic in the torus $T^{n}$, lift to (d-)paths with different end points. The methods from [18] can be applied to $\tilde{X}$ immediately. It is probably easier to get hold on periodicity properties in this setting. This line is currently being investigated by several colleagues; see Fajstrup [7] and Fajstrup et al [8].

\section{Implementation issues}

\subsection{A directed graph associated with a cubical complex}

To a cubical complex $X$, one may associate - forgetting dimensions and the pre-cubical relations - a directed graph $\vec{\Gamma}(X)$ : the vertices are the cubes in $X: V(\vec{\Gamma}(X))=\bigcup_{n} X_{n}$; to every vertex $=$ cube $c \in X_{n}$, we associate $\operatorname{arcs}$ from $c$ to $\partial_{i}^{1} c$ and from $\partial_{i}^{0}$ to $c$. The past $\downarrow c \subset X$ is then the union of all predecessors of $c$ regarded as a vertex in $\vec{\Gamma}(X)$; likewise, its future $\uparrow c \subset X$ is the union of all successors of $c$. Both can be determined recursively. Moreover, for a set $C$ of cells, $\downarrow C=\bigcup_{c \in C} \downarrow c$.

\subsection{Steps in the determination of a trace complex}

In this section, we collect a few ideas on how to start the design of an algorithm determining the complex $\mathbf{T}(X)\left(\mathbf{x}_{0}, \mathbf{x}_{1}\right)$ associated to a nonlooping finite pre-cubical complex $X$ and two vertices $\mathbf{x}_{0}, \mathbf{x}_{1} \in X_{0}$ : 
- The lower corner $L(c) \in X_{0}$ of an $n$-cell $c \in X_{n}$ can be determined as $L(c)=\left(\partial_{0}^{0}\right)^{n}(c)$. Altogether, this recipe defines a map $L: X \rightarrow X_{0}$.

- A maximal cell $c \in X$ has no coface under $\partial_{*}^{0}$; maximal is to be understood with respect to a lower vertex. The set $M(X)$ of maximal cells is thus of the form $M(X)=\bigcup_{n} X_{n} \backslash \bigcup_{0 \leq i \leq n} \partial_{i}^{0}\left(X_{n+1}\right)$. The restriction of the map $L$ to $M(X)$ denoted by $L_{M}: M(X) \rightarrow X_{0}$ associates to a maximal cell $c$ its lower corner vertex in $X_{0}$.

- A branch point $v \in X_{0}$ is characterized by $\left|L_{M}^{-1}(v)\right|>1$. Given the map $L_{M}$, the set of branch points $\mathrm{BP} \subset X_{0}$ corresponds to vertices with more than one inverse image, and the set of branch cells $\mathrm{BC}=L^{-1}(\mathrm{BP}) \subset M(X) \subset X$ corresponds to the union of these inverse images.

Using the directed graph $\vec{\Gamma}(X)$ from Section 6.1, one can determine consecutively

- the pasts $\downarrow c_{i}$ for all branch cells $c_{i} \in \mathrm{BC}$,

- the unions $\bigcup_{L\left(c_{j}\right)=L\left(c_{i}\right) \downarrow c_{j}}$ for every branch point $c_{i} \in \mathrm{BC}$ and their comple-

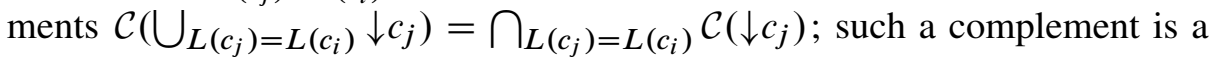
pre-cubical complex since the cells $c_{i}$ are maximal,

- the pre-cubical complex $X_{i}=\downarrow c_{i} \cup \mathcal{C}\left(\bigcup_{L\left(c_{j}\right)=L\left(c_{i}\right)} \downarrow c_{j}\right)$ for every branch cell $c_{i}$.

The next step is the investigation of higher order branch points and branch cells:

- A section $s$ : BP $\uparrow \mathrm{BC}$ fixes one maximal branch cell $c_{j}^{i}$ for every branch point $b_{i} \in \mathrm{BP}$. Form the intersection subcomplexes $X_{s} \subset X$ via intersections of subgraphs of $\vec{\Gamma}(X)$.

- For each of these subcomplexes as point of departure, iterate to determine second order branch points and branches and associated subcomplexes. Iterate to determine higher order ones.

By recursion, we arrive at the set of all - ie, including higher order - branch points and branch cubes and thus to the objects of the category $\mathcal{M}(X)\left(\mathbf{x}_{0}, \mathbf{x}_{1}\right)$; moreover, for every such object $(S, C)$ the associated nonbranching (!) subcomplex $X_{(S, C)} \subset X$.

To find out whether $(S, C)$ is an object of $\mathcal{C}(X)\left(\mathbf{x}_{0}, \mathbf{x}_{1}\right)$, we have to investigate whether there is a d-path from $\mathbf{x}_{0}$ to $\mathbf{x}_{1}$ in $X_{(S, C)}$. Since this space is nonbranching, the future $\uparrow \mathbf{x}_{0}$ of $\mathbf{x}_{0}$ within it has a unique maximal element by Lemma 3.2. It is therefore enough to find out whether $\mathbf{x}_{1}$ is the only maximal vertex in $X_{(S, C)}$ or whether there is at least one other maximal vertex $\mathbf{v}$. Such a "deadlock" vertex $\mathbf{v}$ has no arrow with tail $\mathbf{v}$ in $\vec{\Gamma}\left(X_{(S, C)}\right)$ in that subcomplex. 


\subsection{Final comments}

Although each single of the steps to be taken is quite easy to implement, the number of steps can be enormous. In particular, if higher order branch points arise, the categories $\mathcal{C}(X)\left(\mathbf{x}_{0}, \mathbf{x}_{1}\right) \subseteq \mathcal{M}(X)\left(\mathbf{x}_{0}, \mathbf{x}_{1}\right)$ may be huge, even for a HDA $X$ of moderate size. As in the semaphore case in Raussen [18; 19], it is enough to determine the minimal "dead" objects $(S, C)$ with deadlocks in $X_{(S, C)}$. Still, the determination of the category $\mathcal{C}(X)\left(\mathbf{x}_{0}, \mathbf{x}_{1}\right)$ describing the homotopy type of the trace space of a pre-cubical complex may need a lot of time and memory.

\section{References}

[1] R Brown, P J Higgins, Colimit theorems for relative homotopy groups, J. Pure Appl. Algebra 22 (1981) 11-41 MR621285

[2] R Brown, P J Higgins, On the algebra of cubes, J. Pure Appl. Algebra 21 (1981) 233-260 MR617135

[3] P Bubenik, Simplicial models for concurrency, from: "Proceedings of the workshop on Geometric and Topological Methods in Computer Science”, Electronic Notes in Theoretical Comp. Sci. 283 (2012) 3-12

[4] E W Dijkstra, Co-operating sequential processes, from: "Programming languages", (F Genuys, editor), Academic Press, New York (1968) 43-110

[5] U Fahrenberg, M Raussen, Reparametrizations of continuous paths, J. Homotopy Relat. Struct. 2 (2007) 93-117 MR2369163

[6] L Fajstrup, Dipaths and dihomotopies in a cubical complex, Adv. in Appl. Math. 35 (2005) 188-206 MR2152887

[7] L Fajstrup, Trace spaces of directed tori with rectangular holes, Tech. Report R-2011-08, Department of Math. Sciences, Aalborg University (2011) Available at http://vbn.aau.dk/files/55669400/R_2011_08.pdf

[8] L Fajstrup, E Goubault, E Haucourt, S Mimran, M Raussen, Trace spaces: An efficient new technique for state-space reduction, from: "European Symposium on Programming 2012”, (H Seidl, editor), Lecture Notes in Comp. Sci. 7211, Springer, Berlin (2012) 274-294

[9] L Fajstrup, M Raußen, E Goubault, Algebraic topology and concurrency, Theoret. Comput. Sci. 357 (2006) 241-278 MR2242768

[10] M Farber, Zeros of closed 1-forms, homoclinic orbits and Lusternik-Schnirelman theory, Topol. Methods Nonlinear Anal. 19 (2002) 123-152 MR1921889

[11] M Farber, Topology of closed one-forms, Math. Surveys and Monogr. 108, Amer. Math. Soc. (2004) MR2034601 
[12] M Grandis, Directed homotopy theory, I, Cah. Topol. Géom. Différ. Catég. 44 (2003) 281-316 MR2030049

[13] M Grandis, Directed algebraic topology: Models of non-reversible worlds, New Math. Monogr. 13, Cambridge Univ. Press (2009) MR2562859

[14] D Kozlov, Combinatorial algebraic topology, Algorithms and Computation in Math. 21, Springer, Berlin (2008) MR2361455

[15] V Pratt, Modelling concurrency with geometry, from: "Proc. of the 18th ACM SIGPLAN-SIGACT Symposium on Principles of Programming Languages", (D Wise, editor), ACM, New York (1991) 311-322

[16] M Raussen, Reparametrizations with given stop data, J. Homotopy Relat. Struct. 4 (2009) 1-5 MR2481616

[17] M Raussen, Trace spaces in a pre-cubical complex, Topology Appl. 156 (2009) 17181728 MR2521708

[18] M Raussen, Simplicial models of trace spaces, Algebr. Geom. Topol. 10 (2010) 16831714 MR2683749

[19] M Raussen, Execution spaces for simple higher dimensional automata, Appl. Algebra Engrg. Comm. Comput. 23 (2012) 59-84 MR2917117

[20] C P Rourke, B J Sanderson, $\triangle$-sets I: Homotopy theory, Quart. J. Math. Oxford Ser. 22 (1971) 321-338 MR0300281

[21] D Sullivan, Infinitesimal computations in topology, Inst. Hautes Études Sci. Publ. Math. (1977) 269-331 MR0646078

[22] R J van Glabbeek, Erratum to: "On the expressiveness of higher dimensional automata" [Theoret. Comput. Sci. 356 (2006) 265-290 MR2223695], Theoret. Comput. Sci. 368 (2006) 168-194 MR2274947

[23] G Winskel, M Nielsen, Models for concurrency, from: "Handbook of logic in computer science, Vol. 4", (S Abramsky, D M Gabbay, T S E Maibaum, editors), Oxford Univ. Press, New York (1995) 1-148 MR1365754

Department of Mathematical Sciences, Aalborg University

Fredrik Bajersvej 7G, DK-9220 Aalborg Øst, Denmark

raussen@math.aau.dk

http://people.math.aau.dk/ raussen

Received: 13 September 2011 